\title{
Volumetria de Khaya senegalensis pelo método da altura relativa
}

\author{
Rafaella da Costa Gama ${ }^{1}$ Valdir Carlos Lima de Andrade ${ }^{1}$ \\ ${ }^{1}$ Universidade Federal do Tocantins, Chácara 69-72 Rua Badejos, Lote 7 s/n - Jardim Sevilha, Gurupi - TO, 77404-970 \\ *Author for correspondence: rafaellacg@yahoo.com.br
}

Received: February 2019/ Accepted: September 2019/ Published: September 2019

\section{Resumo}

O Método da Altura Relativa procura promover rapidez e redução de custos ao inventário florestal. Logo, o objetivo do presente trabalho foi analisar o volume estimado por árvore pelo Método da Altura Relativa em comparação ao obtido através da tradicional cubagem rigorosa. Os volumes individuais serão comparados pelo menor erro médio percentual (EMP), menor raiz quadrada do erro médio (RQEM), maior correlação linear múltipla (Ryy (\%)), menor Bias e análise gráfica do resíduo. E foi ainda utilizada a exatidão determinada pelo teste qui-quadrado $\left(\chi^{\wedge} 2\right)$ com nível de significância de 0,05 (P\%). Então, modelos volumétricos foram ajustados com os dados provenientes das metodologias avaliadas, e comparados através coeficiente de determinação ajustado (CDA), menor erro padrão residual (EPR) e análise gráfica dos resíduos. Observou-se então que o Método da Altura Relativa Original foi mais eficiente em estimar o volume individual quando utilizado o coeficiente angular da reta definido pelo i-ésimo intervalo na j-ésima árvore-amostra (CARij). Ao usar o coeficiente angular da reta médio ('CAR_i) o método modificado mostrou ser superior. Quanto ao ajuste dos modelos, os resultados foram semelhantes e satisfatórios, desse modo, é possível afirmar que o Método da Altura Relativa é efetivo para gerar dados que poderiam ser utilizados no ajuste de modelos volumétricos.

Palavras-chave: Volume; Método Geométrico; Geometria Analítica.

\begin{abstract}
The Relative Height Method seeks to promote speed and cost reduction for forest inventory. Therefore, the objective of the present work was to analyze the volume estimated by tree by the Relative Height Method in comparison to the one obtained through the traditional rigorous cubing. The individual volumes will be purchased by the smallest mean error (EMP), the smallest square root of the mean error (RQEM), the highest multiple linear correlation (Ryy (\%)), the smaller Bias and graphical analysis of the residue. And the accuracy determined by the chi-square test $\left(\chi^{\wedge} 2\right)$ with a significance level of $0.05(\mathrm{P} \%)$ was also used. Then, volumetric models were adjusted with the data from the methodologies evaluated, and compared through adjusted determination coefficient (CDA), lower residual standard error (EPR) and graphical analysis of the residues. It was then observed that the Original Relative Height Method was more efficient in estimating the individual volume when using the angular coefficient of the line defined by the i-th interval in the $j$-th sample tree (CARij). When using the angular coefficient of the mean line (-CAR_i) the modified method proved to be superior. Regarding the fit of the models, the results were similar and satisfactory, so it is possible to affirm that the Relative Height Method is effective to generate data that could be used in the adjustment of volumetric models.
\end{abstract}

Keywords: Volume; Geometric Method; Analytical Geometry.

\section{Introdução}

A volumetria de árvores está inserida no cotidiano das pessoas desde os primórdios, decorrente da importância da madeira na vida do homem. (Péllico Neto 2004). No Brasil o setor florestal tem importância relevante na economia, os produtos florestais brasileiros são competitivos no mercado exterior, pois o país possui terras apropriadas para implantação de plantios florestais, as características edafoclimáticas são favoráveis, a idade de corte é menor que em países de clima temperado ou frio e possui tecnologia e infraestrutura adequadas (Silva et al. 2005).

O conhecimento do estoque florestal é imprescindível para o planejamento da condução de plantios florestais a curto, médio e longo prazo, e o procedimento mais comum para conhecer o volume de plantios florestais são as equações de volume, que são geradas com dados prévios de algum método de cubagem rigorosa realizado em árvores selecionadas. Uma metodologia que em geral requer o abate de árvores amostra e medição de vários diâmetros ao longo do fuste, uma atividade onerosa e distante da relativa facilidade de obtenção do diâmetro à altura do peito e altura das árvores (Cabacinha et al. 2013).

O Método da Altura Relativa, que visa promover rapidez e redução de custos ao inventário florestal, caracteriza-se, então, por não ser necessário abater e cubar árvores-amostra. Além disso, as informações, a serem obtidas com a árvore em pé, são medidas apenas para algumas árvores de cada parcela do inventário, cerca de cinco indivíduos (Andrade e Leite 1998; Leite e Andrade 2002). Esse método possui também a vantagem de permitir a realização de medições de diâmetro com a árvore em pé, utilizando-se o Relascópio de Bitterlich ou um Pentaprisma (Scolforo e Thiersch 2004; Leite e Andrade 2004).

Esta metodologia foi desenvolvida por Andrade e Leite (1997), com base nos princípios dos sólidos de revolução mostrados por Gomes (1957), para ter uma sequência de procedimentos à serem empregados nas atividades de quantificação de povoamentos florestais.

Então, através de transformações algébricas no coeficiente angular da reta para intervalos pré-definidos nas árvores em pé, torna-se possível estimar o diâmetro em diferentes alturas, que posteriormente calcular o volume individual com base na forma da árvore, sem o abate dos indivíduos (Cabacinha 2003, Andrade et al. 2009).

O objetivo do presente trabalho foi analisar o volume estimado por árvore pelo método da altura relativa original e modificado, em comparação ao volume obtido pelo processo usual de cubagem rigorosa. E avaliar o ajuste do modelo de Schumacher e Hall Logarítmico (1933) e Soate (1945) utilizando os conjuntos de dados da cubagem rigorosa e os conjuntos obtidos pelo método da altura relativa original e modificado, para a espécie Khay a senegalensis.

\section{Material e Métodos Área de coleta de dados}

A coleta de dados foi conduzida em plantio seminal de mogno africano, da espécie Khaya senegalensis, na Fazenda 
Jarina, zona rural de Gurupi, região sul do estado do Tocantins. A propriedade é localizada na latitude $11^{\circ} 42^{\prime} 42.2^{\prime \prime}$ sul, longitude $48^{\circ} 59^{\prime} 06.8^{\prime \prime}$ oeste, com espaçamento inicial de $6 \times 4$ m e idade de 2,9 anos, a área ocupada pelo povoamento é de 12 hectares.

O clima da região, segundo a classificação de Köppen (1984) enquadra-se em Tropical de savana (Aw), clima tropical com estação seca. A temperatura média anual varia de 28 a $30{ }^{\circ} \mathrm{C}$ e precipitação média anual varia de 1.200 a $1.400 \mathrm{~mm}$, sendo verão chuvoso, inverno seco e elevado déficit hídrico entre os meses de maio a setembro (Inmet, 2017).

Os dados foram coletados em uma área de $7500 \mathrm{~m}^{2}$, que foi dividida em 10 parcelas retangulares de $750 \mathrm{~m}^{2}$ cada (30 x $25 \mathrm{~m}$ ), implantadas aleatoriamente.

Todos os critérios de avaliação e validação foram calculados no Programa R for Windows versão 3.4.4.

\section{Dados coletados}

Foram lançadas, aleatoriamente 10 parcelas retangulares de $750 \mathrm{~m}^{2}$ cada, nessas parcelas, 38 árvores foram cubadas rigorosamente pelo método de Smalian. As medições ao longo do fuste foram realizadas com uma fita métrica de 150 $\mathrm{cm}$, e foram tomados os diâmetros nas posições: 0,$10 ; 0,30$; 0,$70 ; 1,30 ; 2,00$; e seguindo sucessivamente com intervalos de um em um metro até a altura total.

\section{Método da altura relativa original}

Para estimar os volumes individuais das árvores pelo Método da Altura Relativa Original, desenvolvido por Andrade e Leite (1997), foi medida a altura total (ht) de cada árvore, os diâmetros do fuste nas alturas $0,1 \mathrm{~m}\left(\mathrm{~h}_{0,1}\right)$, e a 1,3 $\mathrm{m}$ do solo $\left(\mathrm{h}_{1,3}\right)$. Foi calculada a altura relativa $(\mathrm{hr})$ de cada árvore, a partir da expressão 1 .

$$
h r=\frac{(H t-2)}{2}(1)
$$

Utilizando os dados coletados, foram obtidos os Coeficientes Angulares das Retas (CAR), para três diferentes intervalos do fuste (Intervalo $\mathrm{I}=0,1 \leq \mathrm{h}_{\mathrm{ij}} \leq 1,30$; Intervalo $\mathrm{II}=$ $1,30<\mathrm{h}_{\mathrm{ij}} \leq \mathrm{hr}$; Intervalo III $=\mathrm{hr}<\mathrm{h}_{\mathrm{ij}} \leq \mathrm{ht}$ ), aplicando-se as expressões:

$$
\begin{gathered}
C A R_{I j}=\frac{1,3-h_{0,1}}{\left(\frac{D A P j-d_{0,1}}{2}\right)}(2) \\
C A R_{I I j}=\frac{1,3-h r_{j}}{\left(\frac{D A P_{j}-d_{h r_{j}}}{2}\right)}(3) \\
C A R_{I I I j}=\frac{h r_{j}-h t_{j}}{\frac{d_{h r_{j}}}{2}}(4)
\end{gathered}
$$

Em que:

$\mathrm{CAR}_{\mathrm{ij}}=$ Coeficiente angular da reta definido pelo i-ésimo intervalo na j-ésima árvore amostra, em que i = I, II e III;

$\mathrm{d}_{0,1}, \mathrm{DAP}_{\mathrm{j}}$ e $\mathrm{d}_{\mathrm{hrj}}=$ Diâmetros medidos à $\mathrm{h}_{0,1} ; \mathrm{h}_{1,3} \mathrm{e} \mathrm{hr}$ metros do nível do terreno, na j-ésima árvore amostra;

$\mathrm{ht}_{\mathrm{j}}=$ Altura total da j-ésima árvore amostra.

Os coeficientes angulares das retas geraram as equações taper 5, 6 e 7, que possibilitaram estimar os diâmetros nos três intervalos distintos do fuste:

$$
\begin{gathered}
d_{(0,1 \leq \mathrm{hij} \leq 1,30)}=\frac{2 h_{i j}-2,6}{C A R_{I j}}+D A P_{j}(5) \\
d_{(1,30<\mathrm{hij} \leq \mathrm{hr})}=\frac{2 h_{i j}-2,6}{C A R_{I I j}}+D A P_{j}(6) \\
d_{(\mathrm{hr}<\mathrm{hij} \leq \mathrm{ht})}=\frac{h_{i j}-1,3}{C A R_{I I j}}+\frac{h_{i j}-h t_{j}}{C A R_{I I I j}}+\frac{D A P_{j}}{2}(7)
\end{gathered}
$$

Em que:

d $\left(0,1 \leq h_{i j} \leq 1,30\right)=$ Diâmetro na i-ésima altura desejada entre $0,1 \mathrm{~m}$ e $1,30 \mathrm{~m}$ na j-ésima árvore amostra;

$\mathrm{d}\left(1,30<\mathrm{h}_{\mathrm{ij}} \leq \mathrm{hr}\right)=$ Diâmetro na i-ésima altura desejada entre 1,30 m e hr na j-ésima árvore amostra;

$\mathrm{d}\left(\mathrm{hr}<\mathrm{h}_{\mathrm{ij}} \leq \mathrm{ht}\right)=$ Diâmetro na i-ésima altura desejada entre hr e ht na j-ésima árvore amostra.

Ao utilizar as fórmulas $\mathbf{5}$, 6 e 7 os diâmetros foram estimados em várias alturas da árvore de acordo com seus intervalos. Estimados os diâmetros, os volumes individuais das árvores foram calculados aplicando-se sucessivamente a fórmula de Smalian (Cabacinha 2003; Oliveira 2011).

\section{Método da altura relativa modificado}

No Método da Altura Relativa Modificado mantiveramse as medições dos mesmos diâmetros do método original que são nas posições de $0,1 \mathrm{~m}, 1,3 \mathrm{~m}$ (DAP); altura total (ht) e um ponto entre o DAP e ht, chamada de altura relativa (hr) obtida pela expressão 1 . Porém, além dessa hr foram calculadas três novas $\mathrm{hr}$ definidas pelas expressões (Andrade 2001):

$$
\begin{gathered}
h r_{1}=\frac{(h t-1,7)}{1,7}(8) \\
h r_{2}=\frac{(h t-1,4)}{1,4}(9) \\
h r_{3}=\frac{(h t-1,1)}{1,1}(10)
\end{gathered}
$$

Com os dados coletados foram calculados os valores dos Coeficientes Angulares da Reta (CAR) utilizados no método original, além dos quais foram calculados dois novos CAR definidos pelas expressões:

$$
\begin{aligned}
C A R_{I V j} & =\frac{\left(2\left(h r_{1}\right)_{j}-2\left(h r_{2}\right)_{j}\right)}{\left(\left(d_{h r 1}\right)_{j}-\left(d_{h r 2}\right)_{j}\right)}(11) \\
C A R_{V j} & =\frac{\left(2\left(h r_{3}\right)_{j}-2 h t_{j}\right)}{\left(d h r_{3}\right)_{j}}(12)
\end{aligned}
$$

Para cálculo do taper entre $0,1 \leq \mathrm{h}_{\mathrm{ij}} \leq 1,30$ e $1,30<\mathrm{h}_{\mathrm{ij}} \leq \mathrm{hr} \mathrm{r}_{1}$ utiliza-se a mesma formulação do método original.

Para $\mathrm{hr}_{1}<\mathrm{h}_{\mathrm{ij}} \leq \mathrm{hr}_{3}$ :

$$
\begin{aligned}
& d_{\mathrm{hr} 1<\mathrm{hij} \leq \mathrm{hr} 3}=\left(h r_{1}-\right. \\
& \left.h r_{2}\right)_{j}^{-1}\left[\frac{h r_{1}\left(h r_{2}-2 h r_{1}+2 h_{i}+1,3\right)-h_{i}\left(h r_{2}+1,3\right)}{2 C A R_{I I}}+\right. \\
& \left.\frac{\left(h r_{2}-h t\right)\left(h r_{1}-h_{i}\right)}{2 C A R_{I I I}}+\frac{D A P_{j}\left(h_{i}-h r_{1}\right)}{4}\right]+\frac{\left(h_{i}+h r_{1}-2,6\right)_{j}}{\left(C A R_{I I}\right)_{j}}+ \\
& D A P_{j}(13)
\end{aligned}
$$

Para $\mathrm{hr}_{3}<\mathrm{h}_{\mathrm{ij}} \leq \mathrm{h}_{\mathrm{t}}$ : 


$$
\begin{aligned}
& d_{\mathrm{hr} 3<\mathrm{hij} \leq \mathrm{ht}}=\left[\frac{\left(h_{i}-h t\right)}{\left(h r_{3}-h t\right)}\right]_{j}\left\{\left(h r_{1}-\right.\right. \\
& \left.h r_{2}\right)_{j}^{-1}\left[\frac{h r_{1}\left(h r_{2}-2 h r_{1}+2 h r_{3}+1,3\right)_{-}\left(h r_{3}\left(h r_{2}+1,3\right)\right.}{2 C A R_{I I}}+\right. \\
& \left.\frac{\left(h r_{2}-h t\right)\left(h r_{1}-h r_{3}\right)}{2 C A R_{I I I}}+\frac{D A P_{j}\left(h r_{3}-h r_{1}\right)}{4}\right]+\frac{\left(h r_{3}+h r_{1}-2,6\right)_{j}}{\left(C A R_{I I}\right)_{j}}+ \\
& \left.D A P_{j}\right\}(14)
\end{aligned}
$$

Ao utilizar as fórmulas $5, \mathbf{6}, 13$ e 14, os diâmetros foram estimados em várias alturas da árvore de acordo com seus intervalos. Estimados os diâmetros, os volumes individuais das árvores foram calculados aplicando-se sucessivamente a fórmula de Smalian (Cabacinha 2003; Oliveira 2011)

\section{Alternativas de utilização do método da altura relativa}

$\mathrm{O}$ volume individual foi estimado utilizando o Método da Altura Relativa Original e Modificado, então, as estimativas foram compradas a seus correspondentes reais que foram obtidos via cubagem rigorosa, utilizando a metodologia de Smalian.

Em segundo momento, com base nos Coeficientes Angulares da Reta individuais foi obtido um único valor para cada seção, resultado da média aritmética dos valores calculados pelas equações 2,3 e 4 .

$$
\overline{\mathrm{CAR}}_{\mathrm{i}}=\frac{1}{\mathrm{n}} \sum \mathrm{CAR}_{\mathrm{ij}}
$$

Em que:

$\mathrm{n}$ = número de unidades de amostra;

\section{Ajuste de modelos volumétricos}

Com o volume obtido pela cubagem rigorosa, pelo método da altura relativa original e pelo método da altura relativa modificado foram ajustados os modelos de Schumacher e Hall Logarítmico (1933), por sua ampla utilização para estimação volumétrica, e de Stoate (1945), por ser considerado o melhor modelo para área para estimar o volume segundo Gama e Andrade (2018).

Tabela 1. Modelos volumétricos ajustados para K. senegalensis com 2,9 anos de idade no município de Gurupi - TO.

\begin{tabular}{lll}
\hline $\mathbf{N}^{\circ}$ & Modelo Volumétrico & Autor \\
\hline 1 & $(\mathrm{v})=\beta_{0}+\beta_{1}(\mathrm{H})+\beta_{2}(\mathrm{D})^{2}+\beta_{3}\left(\mathrm{D}^{2} \mathrm{H}\right)+\varepsilon$ & Stoate (1945) \\
2 & (Lnv) $=\beta_{0}+\beta_{1}(\mathrm{D})+\beta_{2}(\mathrm{H})+\varepsilon$ & Schumacher e Hall Logarítmico \\
& & (1933) \\
\hline
\end{tabular}

$\beta \mathrm{n}=$ coeficientes a serem estimados, $\mathrm{v}=\operatorname{volume}$ total $\left(\mathrm{m}^{3}\right), \mathrm{H}$ $=$ altura total $(\mathrm{m}), \mathrm{D}=$ diâmetro medido à $1,3 \mathrm{~m}$ do solo $(\mathrm{cm})$, $\mathrm{Ln}=$ logaritmo neperiano.

\section{Critérios de análise}

Os critérios para analisar foram o menor erro médio percentual (EMP), menor raiz quadrada do erro médio (RQEM), maior correlação linear múltipla $(\operatorname{Ryy}(\%))$, menor Bias e gráficos de resíduos. $\mathrm{E}$ foi ainda aplicado o procedimento estatístico de exatidão determinada pelo teste qui-quadrado $\left(\chi^{2}\right)$ com nível de significância de $0,05(\mathrm{P} \%)$ em dados independentes. (Leite e Andrade 2002; Cabacinha 2003; Leite e Andrade 2004; Almeida et al. 2011; Terra et al. 2017).

$$
\mathrm{EMP}=\frac{1}{\mathrm{n}} \sum_{\mathrm{i}=1}^{n}\left[\left(\frac{\mathrm{Yi}_{\mathrm{e}}-\mathrm{Yi}_{\mathrm{o}}}{\mathrm{Yi}_{\mathrm{o}}}\right) 100\right](16)
$$

$$
\begin{aligned}
& \mathrm{RQEM}=\sqrt{\frac{\sum_{\mathrm{i}=1}^{\mathrm{n}}\left(\mathrm{Yi}_{\mathrm{e}}-\mathrm{Yi}_{\mathrm{o}}\right)^{2}}{\mathrm{n}}}(17) \\
& \mathrm{r}_{\mathrm{Y}_{0} \mathrm{Y}_{\mathrm{e}} \%}= \\
& {\left[\frac{\sum_{\mathrm{i}=1}^{\mathrm{n}} \mathrm{Yi}_{\mathrm{o}} \mathrm{Yi}_{\mathrm{e}}-\frac{\left.\left(\sum_{\mathrm{i}=1}^{\mathrm{n}} \mathrm{Yi}_{\mathrm{o}}\right) \sum_{\mathrm{i}=1}^{\mathrm{n}} \mathrm{Yi}_{\mathrm{e}}\right)}{\mathrm{n}}}{\sqrt{\left.\left\{\sum_{\mathrm{i}=1}^{\mathrm{n}}\left(\mathrm{Yi}_{\mathrm{o}}-\mathrm{Ym}\right)^{2}\right]\left[\sum_{\mathrm{i}=1}^{\mathrm{n}}\left(\mathrm{Yi}_{\mathrm{e}}-\mathrm{Ym}\right)^{2}\right]\right\}}}\right] 10} \\
& \operatorname{Bias}_{\%}=\frac{\left(\sum_{\mathrm{i}=1}^{\mathrm{n}} \mathrm{Yi}_{\mathrm{e}}-\sum_{\mathrm{i}=1}^{\mathrm{n}} \mathrm{Yi}_{\mathrm{o}}\right)}{\sum_{\mathrm{i}=1}^{\mathrm{n}} \mathrm{Yi}_{\mathrm{o}}} 100 \\
& \text { Resíduo\% }=\left(\frac{\mathrm{Y}_{o}-\mathrm{Y}_{e}}{\mathrm{Y}_{\mathrm{o}}}\right) * 100 \\
& \mathrm{P} \%=\sqrt{\frac{196^{2}}{\chi_{\mathrm{n}}^{2}} \sum_{\mathrm{i}=1}^{\mathrm{n}}\left(\frac{\mathrm{Yi}_{\mathrm{o}}-\mathrm{Yi}_{\mathrm{e}}}{\mathrm{Yi}_{\mathrm{o}}}\right)^{2}}
\end{aligned}
$$

Em que:

$\mathrm{Yi}_{\mathrm{e}}=$ valor estimado da variável de interesse (volume);

$\mathrm{Yi}_{\mathrm{o}}=$ valor observado da variável de interesse (volume);

$\mathrm{Y}_{\mathrm{m}}=$ valor real médio;

$\chi_{\mathrm{n}}^{2}=$ valor tabelado do qui-quadrado para $\mathrm{n}$ graus de liberdade a $5 \%$.

A análise gráfica da distribuição dos resíduos entre os valores observados e estimados da variável dependente (volume) possibilita a verificação de ocorrências de tendenciosidades.

Para estabelecer as comparações entre os modelos volumétricos ajustados com cada metodologia foram utilizadas as medidas de precisão: coeficiente de determinação ajustado (CDA), menor erro padrão residual (EPR) e análise gráfica dos resíduos defina anteriormente.

$$
\begin{aligned}
& \mathrm{CDA}=1-\left(\frac{\mathrm{n}-1}{n-\mathrm{p}}\right) \cdot\left(\frac{\text { SQres }}{\text { sQtot }}\right)(22) \\
& \mathrm{EPR}=\sqrt{\mathrm{QMres}}(23) \\
& \mathrm{EPR} \%=\frac{\mathrm{EPR}}{\mathrm{Ym}} \times 100(24)
\end{aligned}
$$

Em que:

$\mathrm{p}=$ Números de coeficientes do modelo; SQres = Soma de quadrados do resíduo; SQtot $=$ Soma de quadrados total;

EPR = Erro padrão residual;

QMres = Quadrado médio do resíduo;

$\mathrm{EPR} \%$ = Erro padrão residual em porcentagem;

Ym = valor real médio;

\section{Resultados e Discussão}

\section{Comparação das Estimativas Volumétricas}

Cada árvore-amostra teve seu volume total obtido pela cubagem rigorosa, Método da Altura Relativa Original e Método da Altura Relativa Modificado, utilizando a metodologia de Smalian. Dessa forma, comparou-se diretamente o volume individual de cada metodologia ao obtido via cubagem rigorosa através de análise estatística (Tabela 2).

Nota-se então que o Método da Altura Relativa Original foi mais eficiente para estimar o volume individual, com valores residuais mais próximos de zero, menos viesado e a correlação linear múltipla mais elevada, comparado ao 
Método da Altura Relativa Modificado quando os diâmetros foram estimados utilizando um CARij.

Ao utilizar um $\overline{\mathrm{CAR}}_{\mathrm{i}}$ para todas as árvores-amostra o Método da Altura Relativa Modificado se mostrou superior em todas as estatísticas analisadas, que demonstra menor diferença entre o volume observado e estimado.

Almeida et al. (2011) compararam o Método da Altura Relativa Original e Modificado, também observaram superioridade da metodologia original para estimar o volume de Eucalyptus urophylla aos cinco anos de idade, utilizando o CAR individual.

Tabela 2. Critérios estatísticos para avaliar o Métodos da Altura Relativa Original e Modificado.

\begin{tabular}{cccccc}
\hline Método & EMP & Ryy\% & RQEM\% & Bias\% & P\% \\
\hline Altura Relativa Original (CARij) & 0,496 & 97,988 & 9,249 & 0,417 & - \\
Altura Relativa Modificado $(\mathrm{CARij})$ & 2,108 & 97,099 & 12,928 & 3,059 & - \\
Altura Relativa Original $\left(\overline{\mathrm{CAR}}_{i}\right)$ & $-1,879$ & 98,221 & 9,812 & $-3,644$ & 9,700 \\
Altura Relativa Modificado $\left(\overline{\mathrm{CAR}}_{i}\right)$ & 0,592 & 99,446 & 4,709 & $-0,011$ & 7,403
\end{tabular}

$\mathrm{EMP}=$ erro médio percentual; RQEM = raiz quadrada do erro médio; Ryy $\%=$ correlação linear múltipla; Bias $\%=$ Bias em porcentagem; $\mathrm{P} \%=$ exatidão determinada pelo teste quiquadrado com nível de significância de 0,05 .

Os resultados supracitados demonstraram boas estimativas do volume individual com método da Altura Relativa Original e Modificado, no entanto, com base na Figura 1 é possível afirmar ainda que existe perda de precisão nas estimativas associada a conicidade das árvores de maior diâmetro, percebe-se essa maior dispersão nas árvores de DAP maior que $8 \mathrm{~cm}$.

A redução na precisão das estimativas foi observada também por Andrade et al. (2006), Thiersch et al. (2006) e Almeida et al. (2011), autores que afirmam que por se tratar da estimativa de diâmetros menores os erros observados podem não afetar negativamente a estimativa do volume.
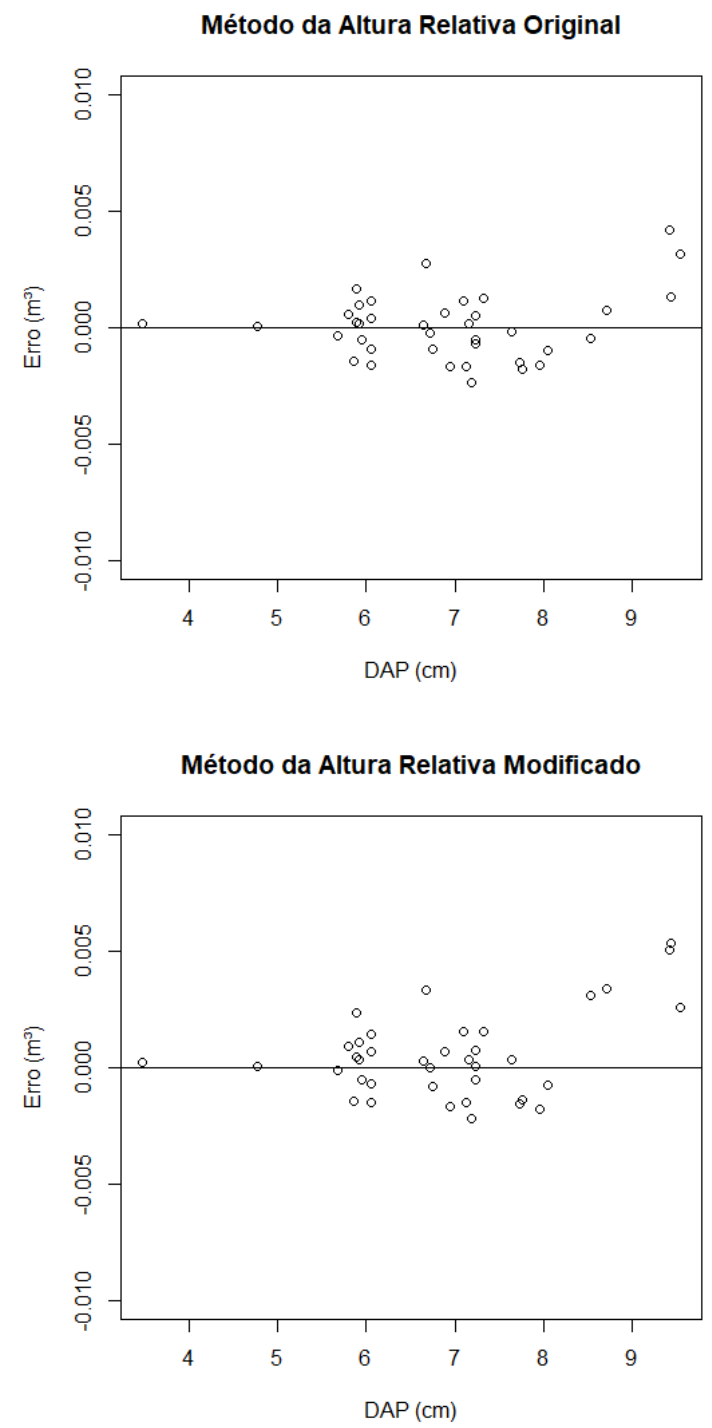

Figura 1. Dispersão dos resíduos do método da altura relativa original e altura relativa modificado.

\section{Avaliação do Ajuste dos Modelos Volumétricos}

Nas Tabelas 3, 4 e 5 observa-se os resultados obtidos com o ajuste dos modelos de Schumacher e Hall (1933) e Stoate (1945) com dados oriundos da cubagem rigorosa, Método da Altura Relativa Original e Modificado, respectivamente. Os resultados foram semelhantes, mas é possível determinar que a metodologia original foi superior as demais, seguida pela cubagem rigorosa e a metodologia modificada.

Cabe ressaltar que a utilização do Método da Altura Relativa Original ou Modificado não demanda o ajuste de modelos matemáticos para estimar o volume, mas os resultados apresentados demonstram que se for necessário os ajustes com dados oriundos dessas metodologias não ocorrerão perdas na qualidade do ajuste. 
Tabela 3. Coeficientes estimados e estatísticas de precisão dos modelos volumétricos ajustados com dados oriundos do método da Altura Relativa Original, Modificado e cubagem rigorosa pelo método de Smalian.

\begin{tabular}{|c|c|c|c|c|c|c|c|}
\hline Método & Modelo & $\widehat{\hat{\beta} 0}$ & $\widehat{\hat{\beta}} 1$ & $\widehat{\bar{\beta}} 2$ & $\overline{\mathrm{\beta} 3}$ & CDA & EPR \\
\hline Altura Relativa & Schumacher e Hall & $-8,958$ & 2,051 & 0,447 & - & 0,948 & 10,76 \\
\hline \multirow[t]{2}{*}{ Original } & Logarítmico (1933) & & & & & & \\
\hline & Stoate (1945) & $4,879^{-3}$ & $-1,046^{-3}$ & $5,318^{-5}$ & $4,831^{-5}$ & 0,949 & 10,30 \\
\hline Altura Relativa & Schumacher e Hall & $-9,029$ & 2,102 & 0,445 & - & 0,923 & 13,19 \\
\hline \multirow[t]{2}{*}{ Modificado } & Logarítmico (1933) & & & & & & \\
\hline & Stoate (1945) & $6,230^{-3}$ & $-1,430^{-3}$ & $2,718^{-5}$ & $5,715^{-5}$ & 0,932 & 12,50 \\
\hline Cubagem & Schumacher e Hall & $-9,069$ & 1,878 & 0,709 & - & 0,929 & 11,39 \\
\hline \multirow[t]{2}{*}{ Rigorosa } & Logarítmico (1933) & & & & & & \\
\hline & Stoate (1945) & $-2,327^{-3}$ & $7,396^{-4}$ & $1,291^{-4}$ & $2,568^{-5}$ & 0,930 & 11,48 \\
\hline
\end{tabular}

$\widehat{\beta}_{0}=$ coeficiente estimado; CDA = coeficiente de determinação ajustado; EPR = erro padrão residual em porcentagem;

Nas Figuras 2, 3 e 4 é constada a semelhança no ajuste dos modelos volumétricos com as três metodologias, fato que corrobora com os resultados das Tabelas 3 .

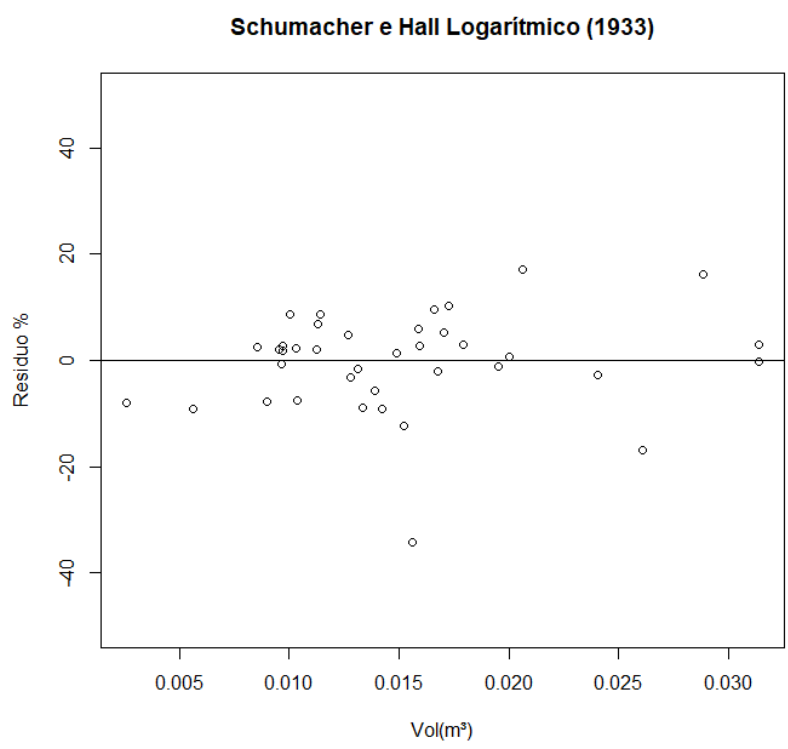

Figura 2. Distribuição de resíduos dos modelos volumétricos ajustados com dados de cubagem rigorosa.
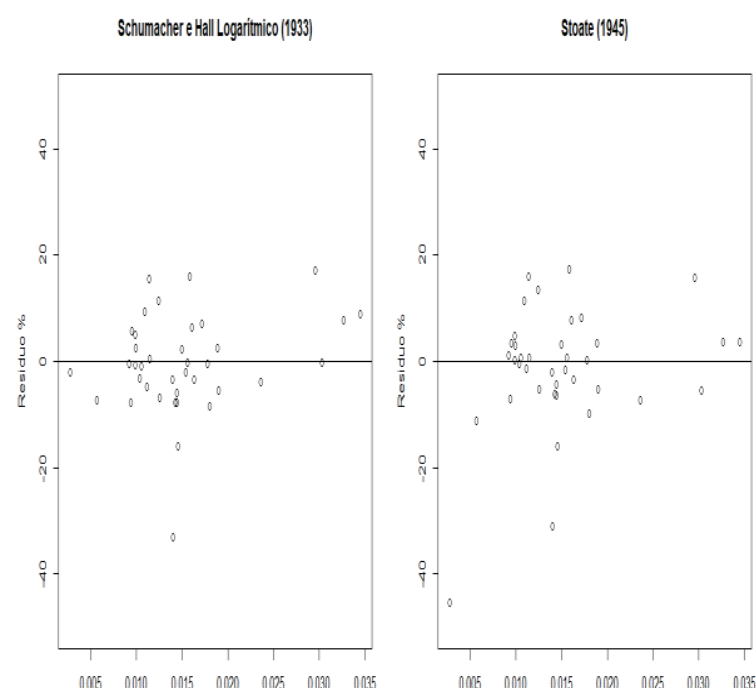

Valmin

Volmin

Figura 3. Distribuição de resíduos dos modelos volumétricos ajustados com dados do método da altura relativa original.
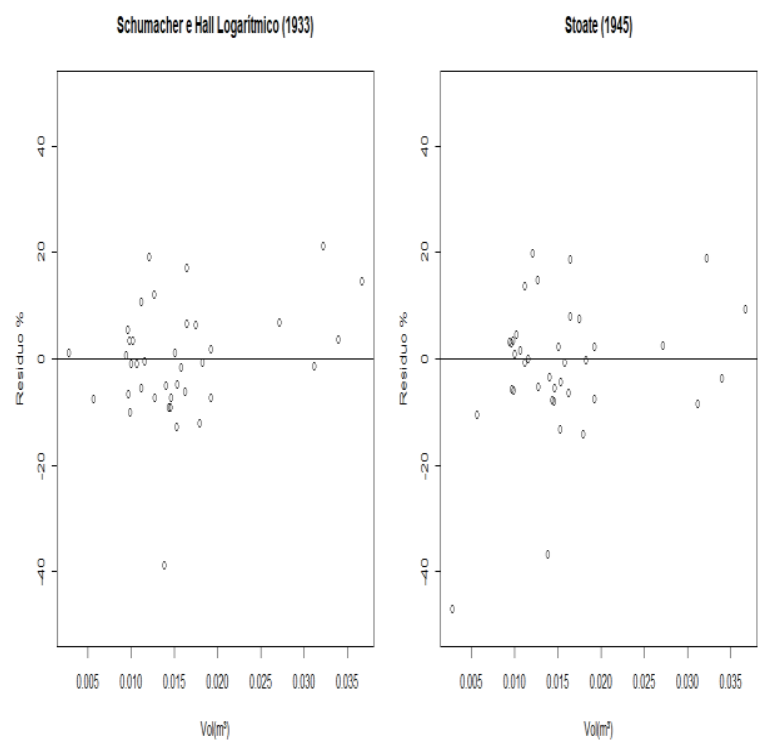

Figura 4. Distribuição de resíduos dos modelos volumétricos ajustados com dados do método da altura relativa modificado.

\section{Conclusão}

As metodologias avaliadas geraram estimativas precisas do volume individual de árvores de Khay a senegalensis de 2,9 anos.

Ambos os métodos são promissores para estimar o volume individual, no entanto, o Método da Altura Relativa Original gerou resultados superiores ao Modificado, quando é empregado nas estimativas dos diâmetros o CAR individual.

Ao utilizar o ${ }^{-}$CAR_i para todas as árvores-amostra a metodologia modificada demonstrou superioridade para estimar o volume individual, com menor diferença entre os valores observados e estimados.

O Método da Altura Relativa demonstrou ser eficiente para obtenção de dados, de forma mais econômica, que 
poderiam ser usados para o ajuste de modelos volumétricos se necessário.

\section{Referências}

Almeida RC, Cabacinha CD, Rocha TB, Paula A (2011) Volumetria de Eucalyptus urophylla S. T. Blake pelo método geométrico do Planalto da Conquista, Bahia. Revista Caatinga, 24 (1): 65-75.

Andrade VCL (2001) Um método para descrever o perfil do tronco em árvores de eucalipto utilizando geometria analítica. Dissertação, Universidade Federal de Viçosa. $74 \mathrm{p}$.

Andrade VCL, Leite HG (1997) Análise do perfil do tronco de árvores utilizando geometria analítica. Iniciação Científica Sem Bolsa de Pesquisa, Universidade Federal de Viçosa. 35p.

Andrade VCL, Leite HG (1998) Uma nova metodologia para medir parcelas em inventários florestais. In: VI Seminário de Iniciação Científica da UFOP, Ouro Preto, Minas Gerais.

Andrade VCL, Calegario N, Scolforo JRS (2006) Análise de algumas alternativas para obter o coeficiente angular da reta no método da altura relativa. Ciência Florestal, 16 (3): 303 317.

Andrade VCL, Calegario N, Souza AL, Rezende JLP, Trugilho PF, Rosado SCS, Silva JRM (2009) Uso de sólidos geométricos para gerar equações de forma do tronco de árvores em pé. Scientia Forestalis, 37 (38): 299-311.

Cabacinha CD (2003) Um método para a realização do inventário florestal suprimindo a cubagem rigorosa. Dissertação, Universidade Federal de Lavras. 166p.

Cabacinha CD, Scolforo JRS, Thiersch CR, Sales NLP, Carvalho LR (2013) Uma nova abordagem para o método geométrico usando o índice da parábola. Ciência Florestal, 23 (1): 261-271.

Gama RC, Andrade VCL (2018) Equações hipsométricas e volumétricas para mogno africano no sul do Tocantins. Scientia Agraria Paranaensis, 17 (4): 507-514.

Gomes AMA (1957) Medição dos arboredos. Lisboa: Livraria Sá da Costa. 413p.

\section{INSTITUTO NACIONAL DE METEOROLOGIA - INMET}

(2017) Disponível em:<
http://www.inmet.gov.br/portal/index.php?r=clima/page\& page $=$ desvioChuvaAnual $>$. Acesso em: 03 de mai.2018.

Leite HG, Andrade VCL (2002) Um método para condução de inventários florestais sem o uso de equações volumétricas. Revista Árvore, 26 (3): 321-328.

Leite HG, Andrade VCL (2004) Uso do método da altura relativa em inventário florestal de um povoamento de Pinus. Revista Árvore, 28 (6): 865-873.

Oliveira CP (2011) Método da altura relativa na estimativa do volume individual para seleção de materiais genéticos de eucalipto. Monografia, Universidade Federal Rural do Rio de Janeiro. 46p.
Péllico Netto S (2004) Equivalência volumétrica: uma nova metodologia para estimativa do volume de árvores. Revista Acadêmica: ciências agrárias e ambientais, 2 (1): 17- 30.

Schumacher FX, Hall FS (1933) Logarithmic expression of timber-tree volume. Journal of Agricultural Research, 47 (9): 719-734

Scolforo JRS, Thiersch CR (2004) Biometria florestal: medição, volumetria e gravimetria. Lavras: UFLA/FAEPE. 285p.

Silva ML, Jacovine, LAG, Valverde SR (2005) Economia Florestal. 2. ed. Viçosa: UFV. 178p.

Stoate IN (1945) The use of a volume equation in pine stands. Australian Forestry, (9): 48-52.

Terra DLCV, Andrade VCL, Ferreira Júnior JM (2017) Funções segmentadas de taper para o clone GG100 no sudeste do Tocantins. Cerrado Agrociências, (8): 104-115.

Thiersch CR, Scolforo JR, Oliveira AD, Maestri R, Dehon G (2006) Acurácia dos métodos para estimativa do volume comercial de clones de Eucaly ptus sp. Cerne, 12 (2): 167 181. 